\title{
Analysis of Motor Imagery EEG Classification Based on Feature Extraction and Machine Learning Algorithm
}

\author{
Rameshwar D. Chintamani ${ }^{1}$, Dr. Parmalik Kumar ${ }^{2}$, Dr. Rajneesh Karan ${ }^{3}$ \\ Ph.D. Scholar ${ }^{1}$, Professor ${ }^{2,3}$, Department of Computer Science \& Engineering ${ }^{1,2,3}$, Madhyanchal Professional \\ University $^{1,2,3}$, Bhopal, Madhya Pradesh, India
}

\begin{abstract}
The brain-computer interface provides the excellent potential to address nervous systemrelated activity. The function of the nervous system work between internal brain control and external human body physical structure. Some parts of the human body cannot generate the signal for the processing of the human brain, cannot recognize and identify human body parts' activity-the motor imagery EEG classification approach helps resolve such types of critical illness cause of death. The dimension and structure of motor imagery-based EEG data are very high and unsupported behaviors. The machine learning and another classification algorithm cannot handle these variants of EEG data directly. For the process of better classification of motor imagery, EEG needs transformation and extraction. The transformbased feature extraction process such as DCT, DWT, SFTF and some other harmonic frequency-based applied. In this paper presents the details analysis of feature extraction and classification algorithms for motor imagery EEG classification. The machine learning provides three types of an algorithm for classification, supervised, unsupervised and semi-supervised. This paper mainly focuses on the supervised machine learning algorithm. For the analysis of machine learning algorithm use $\mathrm{BC}$ competitionIV dataset. MATLAB software is used as a tool for the code of algorithms and measures standard parameters such as accuracy, sensitivity and specificity.
\end{abstract}

Keywords: - BCI, Motor Imagery, EEG, Machine learning, Transform CSP, MATLAB

\section{INTRODUCTION}

The brain-computer interface (BCI) is key components to communicate the human brain to the outside world. Currently, two types of BCI application are used invasive and non-invasive[1]. The non-invasive approach is primarily used due to low cost and convenient operation of the system. Form the operation of non-invasive study and use of signal processing, pattern recognition, medicine, and some others filed. The process of electroencephalogram (EEG) is applied to record brain signals in BCI systems[2, 3, 4]. The format of recoded signal is analog, after recoded signal concerted into digital with the application of $A / D$ converter. The property of recoded signal is nonstationary, these behaviors signal represents terms of time and frequency. The critical illness such as ALS, brain stroke and others serious disease diagnosis with the help of motor imagery EEG classification [5, 6]. The interaction of brain computer interface is in multiple domain such as signal processing, neuroscience and machine learning. In last decade the signal processing and machine learning change the predictive ratio and classification ratio of motor imagery EEG. Various authors proposed machine learning based algorithm for the classification[7]. The diversity of machine learning provides various design methods of classification algorithm such as ensemble, cascading and stacking $[8,9]$. These methods enhance the capacity of motor imagery EEG classification. The entitled literature focus on the extraction of features component of motor imagery EEG classification. The feature extraction is part of pre-processing of EEG data for the process of sampling. The most common dominated feature extraction methods is CSP (common spatial pattern). The CPS feature extraction provide the statical feature component of motor imagery EEG signals. The transform function is another option of feature extraction process [10, $111,12]$. The transform-based function work in domain of time-frequency domain as motor imagery EEG signals. The major dominated applied transform is DWT and CWT[13, 13]. The discrete wavelet transform is derived from the mother wavelet transform. The process of decomposition of discrete transform function derived the new set of feature components of motor imagery-based EEG. Despite the large utilization of CSP feature extractor, the selection of features band in EEG signals is still a challenge. Most of authors work in direction of frequency band selection, which is mainly categorized into four section, CSP combined with time-frequency analysis techniques, orthogonal empirical mode decomposition (OEMD) with FIR filter[14, 15]. Many authors applied various hybrid methods of feature extraction such as combination 
of CSP and wavelet. Further explode the feature extraction process in section III. Potential of classification algorithm depends on the methods of feature selection and feature optimization. The process of features selection and feature optimization deals with swarm-based algorithms. The swarm-based algorithms optimized the artifact and low intensity of signal and improve the rate of classification. Some of authors applied ant colony optimization and particle swarm optimization algorithms for the selection of feature components of EEG signals. This paper mainly focuses on the

\section{RELATED WORK}

The advancement of machine learning increases the ratio of classification in motor imagery-based EEG. The process of advancement deals with contribution of various authors proposed methods. The signal processing also contributes more in scenario of feature extraction and normalization of dataset. The contribution of authors describe here.

Luo, Jing Et al. [1] to planning to improve the presentation of engine symbolism-based EEG classification in a couple channel circumstance, an ESVL-based methodology is talked about to join the benefits of the ERD/ERS-based highlights and the occasion related potential-based highlights in engine symbolism-based EEG classification. ESVL is a troupe learning calculation dependent on help vector machine classifier. Fu, Rongrong Et al. [2] The classification of mental undertakings, for example, engine symbolism dependent on EEG signals is a fundamental issue in the BCI framework. The element extraction is a significant issue for improving classification precision of $\mathrm{BCI}$ framework. For extricating discriminative highlights, CSP is an effective component extraction technique. In any case, highlights separated by CSP are thick, and even component designs are more than once chose in the element space. An inadequate CSP calculation is examined, which installs the meager procedures and iterative inquiry into the CSP. To improve the classification execution, two regularization boundaries are added to the conventional LDA. The scanty CSP calculation can choose a few channels of EEG signals with the clearest highlights. The improved regularized discriminant investigation is utilized to tackle the peculiarity issue and improve the component classification precision. The talked about calculation was assessed by the informational index I of the IVth $\mathrm{BCI}$ rivalry and their dataset. The exploratory aftereffects of the BCI rivalry dataset show that exactness of the improved calculation is $10.75 \%$ higher than that of the customary calculation. Contrasting and the presently existing techniques for a similar information, it additionally shows brilliant classification execution. The effectiveness of the performance of classification algorithm of machine learning. The bottleneck problem of data related finds and convert into challenge and minimize this for the classification of motor imagery EEG classification. The rest of paper organised as in section II. Describe the related work in the area of motor imagery EEG classification. In section III describe the process of feature extraction and machine learning algorithms. In section IV describe the experimental analysis and finally conclude in section $\mathrm{V}$

improved calculation is additionally appeared in analyses on their dataset. Jiao, Yong Et al. [3] A possible limit of a MI based BCI is that it typically requires a generally prolonged stretch of time to record adequate EEG information for vigorous classifier preparing. The adjustment trouble during information procurement stage will most presumably make a subject be hesitant to utilize a BCI framework. To ease this issue, Authors talked about a novel SGRM for improving the effectiveness of MI-based BCI by abusing the between subject data. In particular, gone before by include extraction utilizing normal spatial example, a composite word reference lattice is developed with training tests from both the objective subject and different subjects. By expressly misusing inside gathering scanty and gathering astute meager imperatives, the most conservative portrayal of a test of the objective subject is then assessed as a straight blend of sections in the word reference grid. Characterization is executed by ascertaining the class-explicit portrayal remaining dependent on the critical preparing tests relating to the nonzero portrayal coefficients. Appropriately, the talked about SGRM technique adequately decreases the necessary preparing tests from the objective subject because of assistant information accessible from different subjects. With two public EEG datasets, broad test examinations are completed among SGRM and other cutting edge draws near.

Raza, Haider Et al. [4] a novel combination of CSEUAEL to handle non-stationarity in MI related EEG classification. The talked about strategy first utilizes a dramatically weighted moving normal model to recognize the covariate shifts in the basic spatial example highlights extricated from MI related cerebrum reactions. At that point, a classifier troupe was made and refreshed over the long run to represent changes in streaming info information dissemination wherein new classifiers are added to the outfit as per assessed shifts. Besides, utilizing two freely accessible BCI-related EEG datasets, the examined technique was broadly contrasted and the cutting-edge single-classifier based uninvolved plan, single-classifier based dynamic plan and troupe 
based aloof plans. The test results show that the talked about dynamic plan-based troupe learning calculation significantly upgrades the BCI execution in MI classifications. Taran, Sachin Et al. [5] the AIMFs based highlights are examined for classification of EEG signs of different MI errands. The AIMFs are acquired by applying EMD and Hilbert change on EEG signal. The highlights to be specific: crude snapshot of first subsidiary of momentary recurrence, zone, ghostly snapshot of force phantom thickness, and pinnacle estimation of PSD are figured from AIMFs. The highlights are standardized to diminish the one-sided nature of the classifier. The standardized highlights are applied as contributions to LS-SVM classifier and execution boundaries are figured utilizing different part elements of LS-SVM classifier. Wang, Ping Et al. [6] a classification structure dependent on LSTM organizations. To accomplish powerful classification, a $1 \mathrm{~d}-\mathrm{AX}$ is utilized to extricate compelling sign portrayal for LSTM organizations. Motivated by traditional regular spatial example, channel weighting procedure is additionally sent to improve the adequacy of the talked about classification structure. Public BCI rivalry information are utilized for the assessment of the examined highlight extraction and classification organization, whose exhibition is likewise contrasted and that of the condition of human expressions approaches dependent on other profound organizations.

Tang, Xianlun Et al. [7] to takes the movement imaging EEG signal as the examination object and talked about an inventive semi-managed model called k-SAE. K-SAE searches for the closest neighbour estimations of the examples to develop another info and learns the powerful highlights portrayal by remaking this new contribution rather than the first information, which is not the same as the conventional AE. The Gaussian channel is chosen as the convolution portion work in $\mathrm{k}-\mathrm{SAE}$ to smooth the commotion in the component. Also, the information data and spatial situation of the component map are recorded by max-pooling and un-pooling, that help to forestall loss of significant data. The strategy is applied to two informational indexes for highlight extraction and characterization examinations of engine imaging EEG signals. The exploratory outcomes show that k-SAE accomplishes great acknowledgment precision and outflanks other best in class acknowledgment calculations. Tang, Xianlun Et al. [8] TQWT-based component extraction technique is examined for the classification of various MI undertakings EEG signals. The TQWT boundaries are tuned for the decay of EEG signal into sub-groups. Time area proportions of sub-groups are considered as highlights for MI undertakings EEG signals. The TQWT-put together highlights are tried with respect to least-squares uphold vector machine classifier for the classification of right-hand and right-foot MI undertakings. The talked about strategy gives $96.89 \%$ MI errands classification precision, which is the most noteworthy when contrasted with other existing same informational collection techniques. The recommended technique can be utilized for identification of MI assignments in a BCI framework intended for controlling automated arm and wheel seats, and so on. Baig, Muhammad Zeeshan Et al. [9] Authors present a study of late advancement in filtering channel determination strategies alongside their element extraction and classification techniques for MI-based EEG applications. Khare, Smith K. Et al. [10] MMI empowers correspondence with milieu by estimating cerebrum exercises. The dependability of MMI frameworks is profoundly reliant on the identification of different MI errands. Ideal separation of mind exercises is needed to maintain a strategic distance from miscommunication. EEG signals give a conscientious answer for the improvement of MMI.

Zhang, Li Et al. [11] an ELM based technique is examined to improve the characterization exactness of engine symbolism EEG. The examined strategy develops a group classifier dependent on streamlined ELMs. Molecule swarm streamlining is utilized to at the same time improve the info loads and shrouded inclinations of ELM to stay away from the irregularity and flimsiness of grouping result when ELM utilizes haphazardly created boundaries, and dominant part casting a ballot methodology is utilized to intertwine the order aftereffects of numerous base classifiers to dodge the negative effect of ELM with neighbourhood ideal boundaries on arrangement result. The examined strategy was contrasted and four contending techniques in examinations dependent on two public EEG datasets and some current strategies announced in the writing utilizing the equivalent datasets also. The outcomes show that the talked about technique accomplished huge higher grouping exactness's than those of the contending strategies on both two-class and fourclass engine symbolism information. In addition, contrasted with the current strategies, it actually acquired prevalent normal correctness's of two-class arrangement and performed better for the subjects with moderately helpless exactness's on both twoclass and four-class characterizations. The critical precision improvement shows the predominance of the examined technique. It very well may be a promising contender for exact grouping of engine symbolism EEG in BCI frameworks. Zhang, Yu Et al. [12] Authors talked about a MKELM-based technique for engine symbolism EEG classification. The piece augmentation of ELM favourable to videos a rich method to dodge computation of the shrouded layer yields and inalienably encode it in a 
bit network. Creators examine impacts of two distinctive portion capacities on the exhibition of bit ELM. The MKELM technique is thusly evolved by incorporating these two sorts of portions with a multi-bit learning methodology, which can viably investigate the valuable data from various nonlinear element spaces for heartier classification of EEG. A broad exploratory correlation with two public EEG datasets demonstrates that the MKELM technique

Chaudhary, Shalu Et al. [13] This work presents a procedure dependent on DCNN for MI assignments acknowledgment in the BCI framework. All the more explicitly, the DCNN is utilized for characterization of the RH and RF MI-assignments based EEG signals. The talked about strategy initially changes the information EEG signals into pictures by applying the T-F draws near. The preowned T-F approaches are STFT and CWT. After time recurrence change the pictures of MI-errands EEG signals are applied to the DCNN stage. The pre-prepared DCNN model, AlexNet is investigated for arrangement. The productivity of the talked about strategy is assessed on IVa dataset of BCI rivalry III. The assessment measurements, for example, precision, affectability, particularity, F1score and kappa esteem are utilized for estimating the talked about strategy results quantitatively. The got results show that the CWT approach yields preferable outcomes over the STFT approach. What's more, the examined strategy acquired $99.35 \%$ precision score is the best one among the current strategy's exactness scores. Fu, Rongrong Et al. [14] This work investigates a methodology for single-preliminary MI EEG classification in interpretable bunching. The tensor organized EEG information under $\mathrm{Mu}$ beat is first handled by CSSD to acquire the multi-dimensional CSSD-planned EEG. In the dimensional element decrease, Fisher's proportion is utilized as the expense capacity to consequently find the ideal projection plane with two component vectors of CSSD-planned EEG comparing to the biggest Fisher's proportion. At that point, DRMM that gives a rectangular choice guideline is utilized to recognize ideal element vectors to acknowledge single-preliminary engine symbolism EEG classification in an interpretable manner. This inventive information investigation model produces sensible group results driven by ideal component information. The likelihood thickness dissemination elements of EEG information in two classes can viably clarify the dependability of the rectangular choice principle given by the DRMM. The examined strategy has been approved utilizing the territories under the collector working trademark bend and bunch quality assessment measurements. Exploratory outcomes exhibit its presentation is similar to existing grouping and gives interpretable bunching results when recognizing the engine aim including EEG gives higher order precision than those of the other contending calculations. The trial results affirm that predominance of the discuses MKELM-based technique for exact characterization of EEG related with engine symbolism in BCI applications. their strategy additionally gives a promising and summed up answer for examine the perplexing and nonlinear data for different applications in the fields of master and savvy frameworks.

signals. This paper gives an oddity strategy dependent on interpretable bunching for singlepreliminary MI EEG classification. Furthermore, it might advance the improvement of BCI application.

Belwafi, Kais Et al. [15] a novel DSAA dependent on the least-squares technique is examined to choose the most suitable component extraction and classification calculations couple for each subject. Specifically, the best couple identified during the preparation of the framework is refreshed during internet testing to check the solidness of the chose couple and keep up high framework precision. Broad and efficient examinations were directed on open datasets of 17 subjects in the BCI-rivalry and the outcomes show an improved exhibition for DSAA over other chose best in class techniques. The outcomes show that the examined framework improved the classification exactness for the three picked public datasets by $8 \%$ contrasted with different methodologies. In addition, the talked about framework was effective in choosing the best way notwithstanding the inaccessibility of reference marks. Performing dynamic and self-versatile determination for the best component extraction and classification calculation couple builds the acknowledgment pace of preliminaries notwithstanding the inaccessibility of reference preliminary names. This methodology permits the advancement of a total BCI framework with incredible precision. Chaudhary, Shalu Et al. [16] a whole throughput augmentation issue for a CR helped D2D network is displayed considering the RF-EH system. A joint improvement issue is formed for whole rate boost of cell and D2D clients by considering power distribution, channel task, client blending and transmission time proportion allotment. At that point, the issue is changed into a standard arched enhancement issue subject to control limitations at singular hubs, impedance requirement, and the individual rate. Besides, the mystery limit prerequisites of cell and D2D hubs are additionally thought of. The duality hypothesis is utilized to deteriorate the issue into various subissues and Karush-Kuhn-Tucker conditions are abused to give the arrangement of the sub-issues. The reproduction results are accommodated the approval of their talked about plans.

Tayeb, Zied Et al. [17] a somewhat associated setup in which the choice ought to be made on the parcel 
blends as well as on the arrangement of sending gadgets. To start with, the various occasions happening at every gadget are recognized in order to infer an articulation for the likelihood appropriation of the disentangling delay. The joint advancement issue over the arrangement of communicating gadgets and the bundle mixes of each is, at that point, detailed. The ideal arrangement of the joint improvement issue is determined utilizing a chart hypothesis approach by presenting the collaboration diagram and reformulating the issue as a most extreme weight coterie issue in which the heaviness of every vertex is the commitment of the gadget distinguished by the vertex. Through broad reenactments, the deciphering defers experienced by all gadgets in the PMP design, the FC-D2D arrangement and the PC-D2D setup are thought about. Mathematical outcomes propose that the PCD2D beats the FC-D2D and gives obvious addition particularly to inadequately associated networks. Liu, Minjie Et al. [18] One of the main 5G innovation empowering agents will be D2D correspondences. D2D correspondences comprise a promising method to improve otherworldly, energy and inactivity execution, abusing the actual vicinity of imparting gadgets and expanding asset usage. Moreover, network framework densification has been considered as quite possibly the most significant strategies to build framework execution, exploiting base station vicinity and spatial reuse of framework assets. In any case, could Authors improve framework execution by utilizing both of these two $5 \mathrm{G}$ empowering advances together in a multi-cell climate? How does range share influence execution upgrade. This article examines the ramifications of impedance, densification and range partaking in D2D execution acquire. The in-band D2D approach, where inheritance clients exist together with potential D2D sets, is considered in a multi-cell framework. Overlay and underlay range sharing methodologies are utilized all together for the potential D2D sets to get to the range. Given that two of the most basic issues in the D2D idea are mode choice and client booking, Authors together location them, targeting boosting the absolute framework uplink throughput. In this way, Authors present a radio asset the executive's component for intra-cell and cross-cell overlay/underlay D2D interchanges empowered in a multi-cell framework. Framework level re-enactments are executed to assess the framework execution and look at the patterns of D2D correspondence acquire for the diverse range sharing methodologies and different densification situations. At long last, certifiable SDR-based analyses are performed to test and evaluate D2D interchanges for overlay and underlay range sharing.

Cheng, Dawei Et al. [19] driven by arising Big Data procedures, Authors talked about to plan a major information stage, named D2D Big Data, to empower the remote D2D interchanges among clients viably, to advance substance for suppliers precisely, and to complete offloading insight for administrators proficiently. Creators send a major information stage and further use an enormous scope dataset from a mainstream D2D sharing application, which contains 866 million D2D sharing exercises on 4.5 million documents spread through almost 850 million clients in 13 weeks. By abstracting and investigating multi-dimensional highlights, including on the web practices, content properties, area relations, primary attributes, meeting elements, social arborescence, security protection strategies, etc, Authors confirm and assess the D2D Big Data stage with respect to prescient substance proliferating inclusion. Huang, Zhenhao Et al. [20] Authors first figure the issue of the asset designation among mmWave and the cell band for different D2D sets from the view purpose of game hypothesis. At that point, with the qualities of cell and mmWave correspondences considered, Authors examined an alliance development game to boost the framework total rate in measurable normal sense. Creators additionally hypothetically demonstrate that their examined game meets to a Nash-stable harmony and further arrives at the close ideal arrangement with quick combination rate. Through broad recreations under different framework boundaries, Authors exhibit the predominant presentation of their plan regarding the framework aggregate rate contrasted and a few other pragmatic plans. Venkatachalam, K. Et al. [21] To fulfill the necessities of different arising applications, the future $6 \mathrm{G}$ portable organization is required to be an intrinsically insightful, exceptionally unique, super thick heterogeneous organization that interconnects everything with amazingly low-idleness and fast information transmission. It is accepted that AI will be the most imaginative procedure that can accomplish canny mechanized organization tasks, the board and support in future complex 6G organizations. Driven by AI procedures, D2D correspondence will be one of the bits of the $6 \mathrm{G}$ jigsaw puzzle. Taheri, Samaneh Et al. [22] Mobile interpersonal organizations and D2D correspondences have arisen as promising procedures to help better nearby progressed administrations in 5G organizations. All things considered, the incorporation of versatile interpersonal organizations and D2D interchanges into $5 \mathrm{G}$ organizations acts essential provokes such like how to abuse the social connections of MUs and deal with the obstruction and assets to improve the exhibition of D2D correspondences. To this end, Authors talked about a social-mindful energy efficiency advancement answer for D2D interchanges in 5G organizations. Specifically, Authors first investigate and assess the influence of social connections on the presentation of D2D 
interchanges, which empower us to define the EEO issue while cautiously thinking about both the social connections and actual obstruction between all the MUs. The EEO issue is then addressed for ideal channel mode choice and ideal transmission powers dispensed to every MU to amplify the energy efficiency, by using versatile hereditary calculation. Mathematical outcomes show that contrasted and social-ignorant strategies, their talked about arrangement can accomplish significant improvement regarding energy efficiency and framework throughput while safeguarding the QoS for all clients by considering the range efficiency and transmission power imperatives.

framework EE, framework whole rate, and blackout likelihood for different QoS levels and shifting densities of D2D sets and CUs. Zhang, Zhiwen Et al. [24], a progression of traffic issues, for example, traffic blockage, traffic mishaps, and vehicle exhaust outflow, are progressively hindering city inhabitants, particularly in times of heavy traffic. Perhaps the most overwhelming ways to deal with soothe the traffic clog is to decide the stage timing of traffic signals. Notwithstanding, a significant deficiency of the current stage timing related control techniques is of profoundly computational unpredictability, which causes, somewhat, a reaction delay.

Lee, Byeong-Hoo Et al. [25] Future age organizations will oblige a lot of information traffic and lower inertness. To fulfill these needs, it is crucial for investigate current otherworldly utilize or present new recurrence groups. Presentation of new recurrence groups requires a halfway or complete difference in as of now conveyed framework, which will have high activity use and capital consumption. It is more advantageous to find different arrangements by focusing on gadget related arrangements. One of the answers for accomplish higher spectra efficiency is through D2D correspondence. She, Qingshan Et al. [26] security and protection issues in DD are featured. It is extensive and demonstrates that in-band is far superior to out-band with down to earth and mechanical reasons. To upgrade the extent of the exploration, network level, and framework level $\mathrm{S} \& \mathrm{P}$ issues in the conveyed and brought together frameworks climate with or without focal administration are studied. Alongside a broad review is accommodated the latest work on DD concerning security and protection issues, and correlation among in-band and out-band DD is performed. Ali, Muhammad Ammar Et al. [27] D2D correspondence is quickly advancing into a reasonable strategy for data trade in a cell organization. It has an extremely low start to finish inactivity and can increment ghastly proficiency of a cell organization. The most recent arrivals of $3 \mathrm{GPP}$
Xygonakis, Ioannis Et al. [23] To examines dynamic asset dividing among network substances in a downlink transmission plan to expand EE of the CUs served by either $\mu \mathrm{W}$ or mmWave little cells while keeping a base QoS for the D2D clients. To address this issue, first, a self-versatile force control component for the D2D sets is defined, subject to an obstruction limit for the CUs while fulfilling their base QoS level. In this manner, an EE improvement issue, which is pointed toward boosting the EE for the two CUs and D2D sets, has been addressed. Reenactment results show the viability of their talked about calculation, which examines the innate tradeoffs between

determination have focused on normalize this method of correspondence and coordinate it in the biological system of LTE progressed. This will give more stimulus to the improvement of D2D advances and their appropriation by portable administrators. This paper presents a conversation and basic examination of the primary highlights of D2D correspondence as characterized in Release 12 and ensuing arrivals of $3 \mathrm{GPP}$ determinations.

Mousapour, Leila Et al. [28] D2D correspondence has arisen as a promising innovation for enhancing ghastly proficiency in future cell organizations. D2D exploits the nearness of imparting gadgets for proficient use of accessible assets, improving information rates, diminishing inactivity and expanding framework limit. The examination local area is effectively exploring the D2D worldview to understand its maximum capacity and empower its smooth incorporation into the future cell framework design. Existing studies on this worldview generally center around impedance and asset the board. Creators survey as of late examined arrangements in over investigated and under investigated zones in D2D. These arrangements incorporate conventions, calculations, and structures in D2D. Besides, Authors give new bits of knowledge on open issues in these regions. Chatterjee, Rajdeep Et al. [29] Authors talk about the utilization case situations of D2D correspondence by arranging its applications into two sorts: business and public wellbeing administrations. This is trailed by a top to bottom conversation on the cutting-edge arrangements talked about in different examination considers tending to various issues related with every classification. While examining an enormous number of past works, Authors feature a portion of the open exploration issues and difficulties in D2D interchanges. Padfield, Natasha Et al. [30] This paper clarifies the advantages and difficulties for wise D2D correspondence that will be needed to achieve the prerequisites for IoT. Numerous ventures and normalization bodies have indicated tremendous interest towards the execution of D2D approach in remote organizations. D2D approach 
encourages to work without the fundamental control of the concentrated management which makes remote organizations more range and energy proficient with traffic offloading. Between systems administration gadgets offer different applications towards the improvement of IoT. Actual limitations of the gadgets and asset imperatives of the organizations offer ascent to a few issues which should be settled.

Ha, Kwon-Woo Et al. [31] Authors considered a situation where D2D clients impart within the sight of cell clients in an overlay network arrangement. To examine the income of specialist organizations in money related terms, the paper gives definite articulations of administrator benefit for both D2D and cell clients. All the more explicitly, Authors consider diverse organization boundaries including client thickness, send force and channel varieties to comprehend their effect on the all-out income of the administrator. Xu, Jiacan Et al. [32] Author present advances of SL D2D interchanges as a keyempowering innovation for $5 \mathrm{G}$ eV2X correspondences. Creators give an outline about the asset distribution and booking of various SL D2D modes under in-inclusion and out-of-inclusion application situations. Besides, Authors present the booking for SL D2D V2X interchanges, which depends on SPS as examined inside the 3GPP detail. Reproductions were completed to assess the presentation regarding impact likelihood accepting various estimations of the key boundaries, for example, RRI and asset choice window. Creators at last examine about the open specialized difficulties for super solid and low-dormancy correspondences as refined from the $5 \mathrm{G} \mathrm{V} 2 \mathrm{X}$ use cases presented in 3GPP Rel.15. Zhang, Shaorong Et al. [33] Authors explored PLS for SKGR in D2D interchanges dependent on helpful trusted and non-confided in transfers. By utilizing social ties, Authors abuse three social wonders for secure correspondences, I. e., confided in situation, non-confided in situation and somewhat confided in situation. The alliance game hypothesis is additionally used to choose the ideal hand-off sets for improving SKGR. Based on

of motor imagery EEG signal. The best things in wavelet transform is the best trade-off between temporal and frequency resolution. the wavelet uses finite basis function is called mother wavelet transform[8]. The most of authors designed variety of feature extraction methods based on wavelet transform. The most of extraction methods deal in terms of DWT and CWT and many more derived algorithms for the extraction of features[11]. The function of wavelet derived two property such as entropy and energy of given signal data. So maximum coverage of feature components describes in manners of this. social ties, Authors build up a calculation for SKGR that shields the keys mystery from both busybody and non-confided in chosen transfers. Creators fuse secure transfers choice and framework wide security for D2D interchanges. The security and combination of the examined calculation are likewise demonstrated in their work. Both mathematically and scientifically results confirm adequacy and consistency of their examined plot, which guarantees better SKGR execution in D2D interchanges.

\section{FEATURE EXTRACTION \& MACHINE LEARNING ALGORITHM}

The role of feature extraction in motor imagery EEG classification is very important. The process of feature extraction separates the components of features of raw EEG data[7, 16]. The various authors proposed feature extraction algorithm such CSP (common spatial pattern) filter, STFT, DWT, CWT, AMD and many more ae per requirement of further decompaction of feature components[17]. Some common features extraction methods most of authors are applied describe and discuss here.

\section{(A) COMMON SPATIAL PATTERN (CSP)}

Common spatial pattern filter is most dominated feature extraction methods in case of BCI $[12,18]$. The processing of common spatial pattern deal with maximize the variance of data stemming for the classes. The process of signal represents as signal $\mathrm{S}$ of an EEG sample

$$
\mathrm{S}=\mathrm{WM},
$$

The value of $\mathrm{M}$ is a Nc X T matrix of EEG data and $\mathrm{W}$ is sample value of $\mathrm{L} X \mathrm{Nc}$ represents the projection of CSP. The source of CSP algorithm develop for the two-class problem, further it explodes in multi-class problem extension.

\section{(B) WAVELET TRANSFORM}

Wavelet transform is another approach of feature extraction

\section{(C) ADAPTIVE PARAMETERS (AAR) \\ AUTOREGRESSIVE}

The characteristic of signal describes the process of feature extraction approach. The adaptive autoregressive parameters is another way to extraction process of features components of EEG signals. The various variants are applied for the extraction of feature components. Such model and variants is called $\operatorname{AR}[19,21]$.

\section{MACHINE LEARNING ALGORITHMS}

machine learning provides various algorithms for the process of motor imagery EEG classification. 
The variants of machine learning algorithms such binary classification, regressive classification ensemble classifier, cascading of classifier and hybrid algorithm of classifier. The most of authors focus on two main approach of algorithm is ensemble and hybrid for the enhancement of classification ratio. The SVM (support vector machine) algorithm is behave like regressive and binary classification, work according to the nature of data variants. The main advantage of support vector is large sample size of data processing and reduces the value of outlier. The minimize value of outlier increase the value of classification and prediction. The utility of SVM algorithm is very high in case of motor imagery EEG classification. Some machine learning algorithm describe here[20, 21, 22].

\section{(A) ESVL (ENSEMBLE SUPPORT VECTOR LEARNING)}

The ESVL algorithm designed with the help of support vector machine. The design algorithm increases the size of decision boundary value with maximum interclass margin. The function of probability reduces the possible value of relation with sample data. it also reduces the training sample time for the process of classification. The ESVL algorithm mapped the components of features with different class for the process of classification. The method of ESVL basically focus on the estimation of optimal value of kernel and hyper plan. The value of optimality enhances the capacity of classification[24, 25].

\section{(B) LSTM (LONG SHORT TIME MEMORY) NETWORK}

The long short-term memory (LSTM) network removes the problem of gradients. The advantage of LSTM to incorporate the non-linear, data-dependent manage with the RNN cell. This algorithm applied with CSP feature extractor and enhance the classification ratio of motor imagery EEG classification. The sequence of recurrent neural network(cell) creates different categories of features sample and classify the data of EEG in better way $[23,30]$.

\section{(C) KNN (NEAREST NEIGHBOUR)}

The KNN algorithms surprise with the utilization in the area of biomedical classification. Basically, the KNN classifier is called lazy classifier. The rate of classification of $\mathrm{KNN}$ classifier is $78 \%$. But the nature of variant function of distance equation and measurement of similarity increase the area of $\mathrm{K}$. various authors used as variable selection of features point for the motor imagery EEG classification. Most of ensemble classifier use KNN classifier as booster in process of ensemble classifier[26, 27].

\section{(D) ELM (EXTREME LEARNING MACHINE)}

The extreme learning algorithm is very fast instead of any neural network-based classification algorithm. The author proposed ensemble classifier with ELM algorithm. The ELM learning algorithm is single hidden feedforwarded neural networks (SLFNs). The ELM algorithm reduces the factor of variance and increases the rate of predication and detection of sample data of motor imagery EEG classification[26].

\section{(E) CNN (CONVOLUTIONAL NEURAL} NETWORKS)

The CNN algorithm is part of Deep learning and very appropriate to classification of image data and signal data. The multiple stage of CNN network defines the propagation of feature set of motor imagery EEG signals. The design architecture of $\mathrm{CNN}$ is feedforwarded for the sequence of feature subset. The proposed CNN algorithm along with ensemble classifier and enhance the rate of classification[27].

\section{EXPERIMENTAL ANALYSIS}

To evaluate the performance of proposed algorithm with MALTAB software. The version of software is $\mathrm{R} 2014 \mathrm{a}$ and the configuration of system is I7 processor, 16GB RAM and windows10 operating system. The dataset description already describes in section II[30, 31, 32]. The process of sample of data applied 10 cross fold for the processing of prediction and measurement of parameters. The other two algorithms also apply on dedicated dataset and measure these parameters as given below.

\section{Accuracy

$=\frac{\text { Total No. of Correctly Classified Instances }}{\text { Total No. of Instances }}$

Sensitivity $=\frac{T P}{T P+F N} \times 100$

Specificity $=\frac{T N}{T N+F P} \times 100$

RESULT ANAYSIS

\begin{tabular}{|l|c|c|c|c|c|}
\hline \multicolumn{1}{|c|}{ SUBJECT } & ESVL[1] & LSTM[6] & KNN[7] & ELM[11] & CNN[13] \\
\hline Sub.1 & 56.2 & 58.5 & 57.5 & 64.4 & 81.5 \\
\hline Sub.2 & 74.2 & 75.7 & 78.4 & 80.8 & 73.2 \\
\hline
\end{tabular}




\begin{tabular}{|l|l|l|l|l|l|}
\hline Sub.3 & 89.5 & 91.1 & 78.4 & 82.4 & 87.4 \\
\hline Sub.4 & 69.5 & 70.5 & 74.2 & 75.7 & 70.7 \\
\hline Sub.5 & 85.4 & 88.2 & 89.5 & 91.1 & 84.4 \\
\hline Sub.6 & 66.4 & 70.7 & 69.5 & 70.5 & 75.1 \\
\hline Sub.7 & 84.1 & 84.4 & 85.4 & 88.2 & 66.7 \\
\hline Sub.8 & 77.5 & 75.1 & 78.9 & 79.7 & 81.5 \\
\hline Sub.9 & 68.4 & 66.7 & 70.7 & 71.2 & 73.2 \\
\hline
\end{tabular}

Table 1: Comparison analysis of accuracy using ESVL, LSTM, KNN, ELM and CNN classification with BC4 competition dataset subject's (Sub.1, Sub.2, Sub.3, Sub.4, Sub.5, Sub.6, Sub.7, Sub.8, Sub.9).

\begin{tabular}{|l|c|c|c|c|c|}
\hline \multicolumn{1}{|c|}{ SUBJECT } & ESVL[1] & LSTM[6] & KNN[7] & ELM[11] & CNN[13] \\
\hline Sub.1 & 82.9 & 89.1 & 78.3 & 77.4 & 75.9 \\
\hline Sub.2 & 75.6 & 84 & 88.2 & 85.4 & 84.6 \\
\hline Sub.3 & 84.3 & 86.6 & 78.5 & 89.1 & 78.3 \\
\hline Sub.4 & 78 & 81.1 & 73.8 & 84 & 88.2 \\
\hline Sub.5 & 80.7 & 83.9 & 84.3 & 86.6 & 78.5 \\
\hline Sub.6 & 80.2 & 75.1 & 78 & 81.1 & 73.8 \\
\hline Sub.7 & 84.5 & 85.2 & 88 & 90.9 & 75.9 \\
\hline Sub.8 & 78.8 & 76.3 & 78.2 & 80.6 & 84.6 \\
\hline Sub.9 & 76.4 & 75.5 & 73.5 & 78.4 & 78.3 \\
\hline
\end{tabular}

Table 2: Comparison analysis of sensitivity using ESVL, LSTM, KNN, ELM and CNN classification with BC4 competition dataset subject's (Sub.1, Sub.2, Sub.3, Sub.4, Sub.5, Sub.6, Sub.7, Sub.8, Sub.9).

\begin{tabular}{|l|c|c|c|c|c|}
\hline SUBJECT & ESVL[1] & LSTM[6] & KNN[7] & ELM[11] & CNN[13] \\
\hline Sub.1 & 78.2 & 80.6 & 84.6 & 77.4 & 75.9 \\
\hline Sub.2 & 73.5 & 78.4 & 78.3 & 85.4 & 84.6 \\
\hline Sub.3 & 81.1 & 88.8 & 82.9 & 89.1 & 78.3 \\
\hline Sub.4 & 83.4 & 78.3 & 75.6 & 84 & 88.2 \\
\hline Sub.5 & 80.7 & 83.9 & 84.3 & 86.6 & 78.5 \\
\hline Sub.6 & 80.2 & 75.1 & 78 & 81.1 & 73.8 \\
\hline Sub.7 & 84.5 & 85.2 & 88 & 90.9 & 75.9 \\
\hline Sub.8 & 78.8 & 76.3 & 78.2 & 80.6 & 84.6 \\
\hline Sub.9 & 76.4 & 75.5 & 73.5 & 78.4 & 78.3 \\
\hline
\end{tabular}

Table 2: Comparison analysis of specificity using ESVL, LSTM, KNN, ELM and CNN classification with BC4 competition dataset subject's (Sub.1, Sub.2, Sub.3, Sub.4, Sub.5, Sub.6, Sub.7, Sub.8, Sub.9).

PERFORMANCE ANALYSIS 


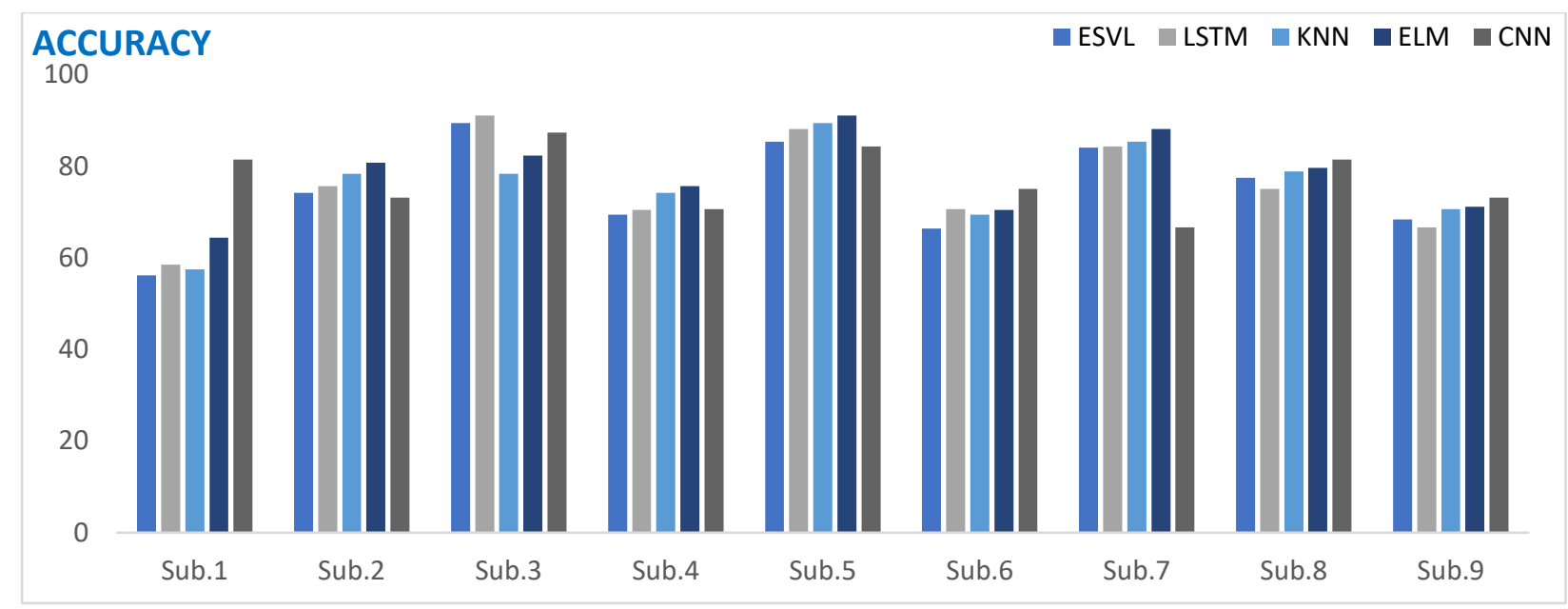

Figure 1: Comparison performance analysis of accuracy used techniques are ESVL[1], LSTM[6], KNN[7], ELM[11] and CNN[13] classification with BC4 competition dataset subject 1 to subject 9.

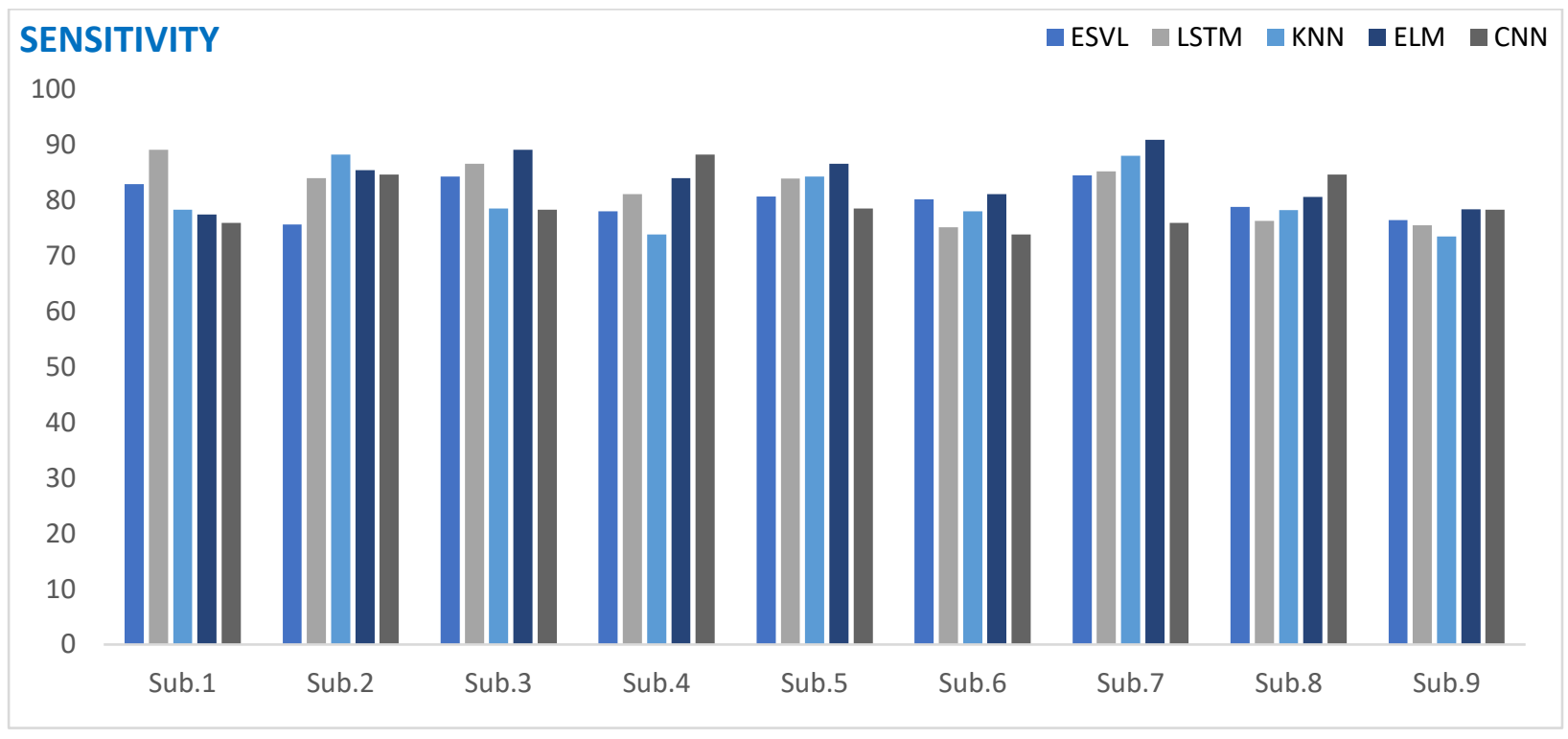

Figure 2: Comparison performance analysis of sensitivity used techniques are ESVL[1], LSTM[6], KNN[7], ELM[11] and CNN[13] classification with BC4 competition dataset subject 1 to subject 9. 


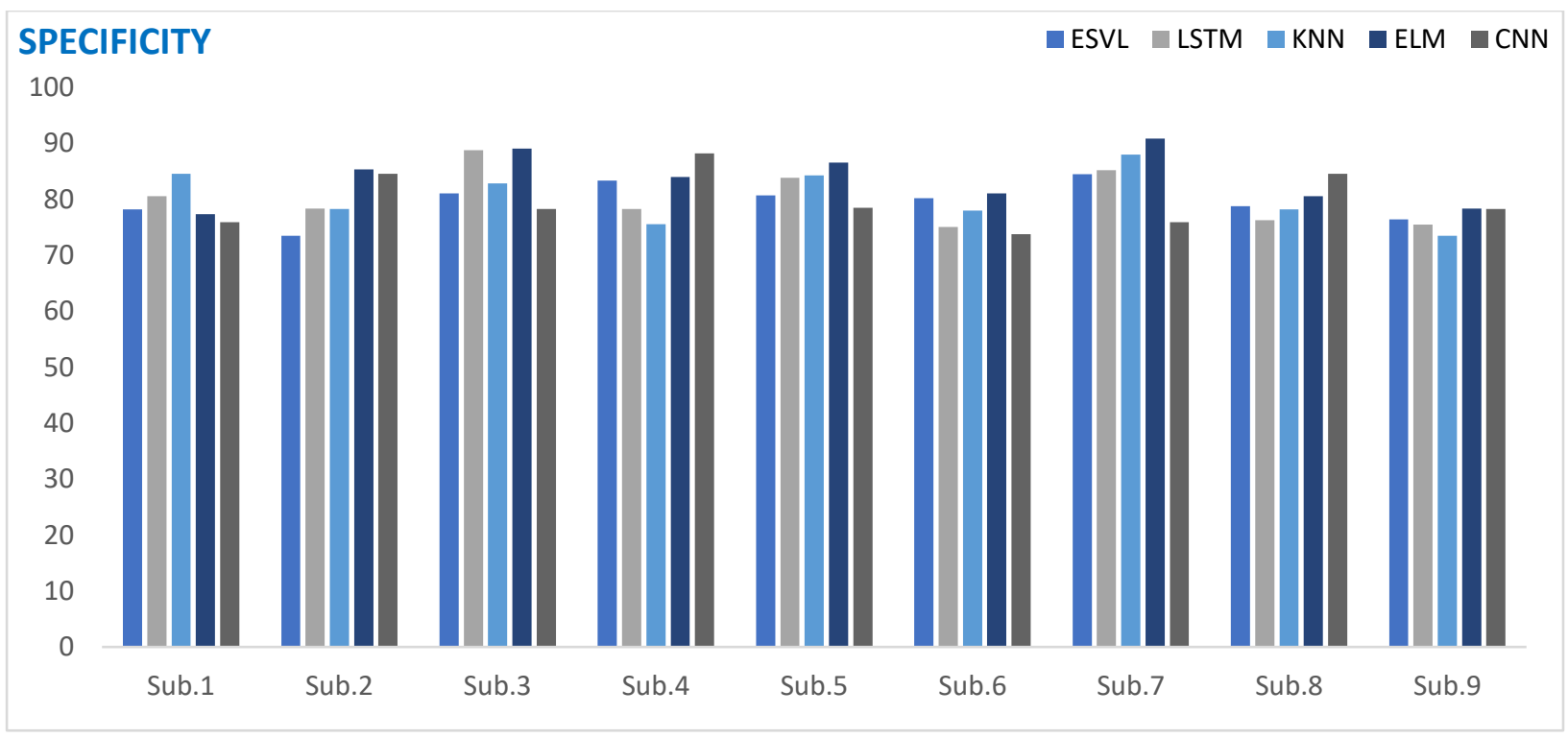

Figure 3: Comparison performance analysis of specificity used techniques are ESVL[1], LSTM[6], KNN[7], ELM[11] and CNN[13] classification with BC4 competition dataset subject 1 to subject 9 .

\section{CONCLUSION \& FUTURE SCOPE}

To evaluate the performance of motor imagery EEG classification based on machine learning algorithm and feature extraction methods. The analysis of results concludes that the better feature selection produces good classification ratio. Different feature selector methods such as CSP, wavelet, ARM and some other methods based on the different approach applied classification algorithms according to their feature selection methods. The feature selection methods algorithm improves the classification ratio and result. This paper mainly focused on and analyzed five classification algorithms of motor imagery such as ESVL, LSTM, KNN, ELM, and CNN. The variation of all these algorithms suggests that the classification target of motor imagery EEG classification is always challenging due to the complex data structure. But the processing of the algorithm rich the capacity of the classification algorithm. In these algorithms, CNN and ELM is the family of neural network algorithms. However, the performance and execution of CNN and ELM are very fast in compression of the conventional network model. The CNN algorithm designed for image and signal data classification. The analysis of results also indicates the gap of feature selection and optimization process. In a survey study, various feature optimization cum selection algorithms for motor imagery EEG classification. Some challenge remains in the process of motor imagery classification. In future proposed hybrid feature selector methods along with ensemble-based classifier.

\section{REFERENCES}

Copyright $\odot$ Authors
[1]. Luo, Jing, Xing Gao, Xiaobei Zhu, Bin Wang, $\mathrm{Na} \mathrm{Lu}$, and Jie Wang. "Motor imagery EEG classification based on ensemble support vector learning." Computer methods and programs in biomedicine 193 (2020): 105464.

[2]. Fu, Rongrong, Mengmeng Han, Yongsheng Tian, and Peiming Shi. "Improvement motor imagery EEG classification based on sparse common spatial pattern and regularized discriminant analysis." Journal of Neuroscience Methods 343 (2020): 108833.

[3]. Jiao, Yong, Yu Zhang, Xun Chen, Erwei Yin, Jing Jin, Xingyu Wang, and Andrzej Cichocki. "Sparse group representation model for motor imagery EEG classification." IEEE journal of biomedical and health informatics 23 , no. 2 (2018): 631-641.

[4]. Raza, Haider, Dheeraj Rathee, Shang-Ming Zhou, Hubert Cecotti, and Girijesh Prasad. "Covariate shift estimation based adaptive ensemble learning for handling non-stationarity in motor imagery related EEG-based braincomputer interface." Neurocomputing 343 (2019): 154-166.

[5]. Taran, Sachin, Varun Bajaj, Dheeraj Sharma, Siuly Siuly, and A. Sengur. "Features based on analytic IMF for classifying motor imagery EEG signals in BCI applications." Measurement 116 (2018): 68-76.

[6]. Wang, Ping, Aimin Jiang, Xiaofeng Liu, Jing Shang, and Li Zhang. "LSTM-based EEG classification in motor imagery tasks." IEEE transactions on neural systems and rehabilitation engineering 26, no. 11 (2018): 2086-2095.

[7]. Tang, Xianlun, Ting Wang, Yiming Du, and Yuyan Dai. "Motor imagery EEG recognition with KNN-based smooth auto- 
encoder." Artificial intelligence in medicine 101 (2019): 101747.

[8]. Tang, Xianlun, Ting Wang, Yiming Du, and Yuyan Dai. "Motor imagery EEG recognition with KNN-based smooth autoencoder." Artificial intelligence in medicine 101 (2019): 101747.

[9]. Baig, Muhammad Zeeshan, Nauman Aslam, and Hubert PH Shum. "Filtering techniques for channel selection in motor imagery EEG applications: a survey." Artificial intelligence review 53, no. 2 (2020): 1207-1232.

[10]. Khare, Smith K., and Varun Bajaj. "A facile and flexible motor imagery classification using electroencephalogram signals." Computer Methods and Programs in Biomedicine 197 (2020): 105722.

[11]. Zhang, Li, Dezhong Wen, Changsheng Li, and Rui Zhu. "Ensemble classifier based on optimized extreme learning machine for motor imagery classification." Journal of neural engineering 17, no. 2 (2020): 026004.

[12]. Zhang, Yu, Yu Wang, Guoxu Zhou, Jing Jin, Bei Wang, Xingyu Wang, and Andrzej Cichocki. "Multi-kernel extreme learning machine for EEG classification in braincomputer interfaces." Expert Systems with Applications 96 (2018): 302-310.

[13]. Chaudhary, Shalu, Sachin Taran, Varun Bajaj, and Abdulkadir Sengur. "Convolutional neural network based approach towards motor imagery tasks EEG signals classification." IEEE Sensors Journal 19, no. 12 (2019): 4494-4500.

[14]. Fu, Rongrong, Weishuai Li, Junxiang Chen, and Mengmeng Han. "Recognizing single-trial motor imagery EEG based on interpretable clustering method." Biomedical Signal Processing and Control 63 (2021): 102171.

[15]. Belwafi, Kais, Sofien Gannouni, Hatim Aboalsamh, Hassan Mathkour, and Abdelfattah Belghith. "A dynamic and self-adaptive classification algorithm for motor imagery EEG signals." Journal of neuroscience methods 327 (2019): 108346.

[16]. Chaudhary, Shalu, Sachin Taran, Varun Bajaj, and Siuly Siuly. "A flexible analytic wavelet transform based approach for motorimagery tasks classification in BCI applications." Computer methods and programs in biomedicine 187 (2020): 105325.

[17]. Tayeb, Zied, Juri Fedjaev, Nejla Ghaboosi, Christoph Richter, Lukas Everding, Xingwei $\mathrm{Qu}$, Yingyu $\mathrm{Wu}$, Gordon Cheng, and Jörg Conradt. "Validating deep neural networks for online decoding of motor imagery movements from EEG signals." Sensors 19, no. 1 (2019): 210 .
[18]. Liu, Minjie, Mingming Zhou, Tao Zhang, and Naixue Xiong. "Semi-supervised learning quantization algorithm with deep features for motor imagery EEG Recognition in smart healthcare application." Applied Soft Computing 89 (2020): 106071.

[19]. Cheng, Dawei, Ye Liu, and Liqing Zhang. "Exploring motor imagery EEG patterns for stroke patients with deep neural networks." In 2018 IEEE International Conference on Acoustics, Speech and Signal Processing (ICASSP), pp. 2561-2565. IEEE, 2018.

[20]. Huang, Zhenhao, Yichun Qiu, and Weijun Sun. "Recognition of motor imagery EEG patterns based on common feature analysis." Brain-Computer Interfaces (2020): $1-9$.

[21]. Venkatachalam, K., A. Devipriya, J. Maniraj, M. Sivaram, A. Ambikapathy, and S. Amiri Iraj. "A novel method of motor imagery classification using eeg signal." Artificial intelligence in medicine 103 (2020): 101787.

[22]. Taheri, Samaneh, Mehdi Ezoji, and Sayed Mahmoud Sakhaei. "Convolutional neural network based features for motor imagery EEG signals classification in brain-computer interface system." SN Applied Sciences 2, no. 4 (2020): 1-12.

[23]. Xygonakis, Ioannis, Alkinoos Athanasiou, Niki Pandria, Dimitris Kugiumtzis, and Panagiotis D. Bamidis. "Decoding motor imagery through common spatial pattern filters at the EEG source space." Computational intelligence and neuroscience 2018 (2018).

[24]. Zhang, Zhiwen, Feng Duan, Jordi SoleCasals, Josep Dinares-Ferran, Andrzej Cichocki, Zhenglu Yang, and Zhe Sun. "A novel deep learning approach with data augmentation to classify motor imagery signals." IEEE Access 7 (2019): 15945-15954.

[25]. Lee, Byeong-Hoo, Ji-Hoon Jeong, and Seong-Whan Lee. "SessionNet: Feature similarity-based weighted ensemble learning for motor imagery classification." IEEE Access 8 (2020): 134524-134535.

[26]. She, Qingshan, Kang Chen, Yuliang Ma, Thinh Nguyen, and Yingchun Zhang. "Sparse representation-based extreme learning machine for motor imagery EEG classification." Computational intelligence and neuroscience 2018 (2018).

[27]. Ali, Muhammad Ammar, Duygu Üçüncü, Pınar Karadayı Ataş, and Süreyya ÖzöğürAkyüz. "Classification of motor imagery task by using novel ensemble pruning approach." IEEE Transactions on Fuzzy Systems 28, no. 1 (2019): 85-91.

[28]. Mousapour, Leila, Fateme Agah, Soorena Salari, and Marzieh Zare. "A Novel Approach to Classify Motor-Imagery EEG with 
Convolutional Neural Network Using Network Measures." In 2018 4th Iranian Conference on Signal Processing and Intelligent Systems (ICSPIS), pp. 43-47. IEEE, 2018.

[29]. Chatterjee, Rajdeep, Ankita Datta, and Debarshi Kumar Sanyal. "Ensemble learning approach to motor imagery EEG signal classification." In Machine Learning in BioSignal Analysis and Diagnostic Imaging, pp. 183-208. Academic Press, 2019.

[30]. Padfield, Natasha, Jaime Zabalza, Huimin Zhao, Valentin Masero, and Jinchang Ren. "EEG-based brain-computer interfaces using motor-imagery: Techniques and challenges." Sensors 19, no. 6 (2019): 1423.

[31]. Ha, Kwon-Woo, and Jin-Woo Jeong. "Motor imagery EEG classification using capsule networks." Sensors 19, no. 13 (2019): 2854.

[32]. Xu, Jiacan, Hao Zheng, Jianhui Wang, Donglin Li, and Xiaoke Fang. "Recognition of EEG signal motor imagery intention based on deep multi-view feature learning." Sensors 20, no. 12 (2020): 3496.

[33]. Zhang, Shaorong, Zhibin Zhu, Benxin Zhang, Bao Feng, Tianyou Yu, and Zhi Li. "The CSP-Based New Features Plus Non-Convex Log Sparse Feature Selection for Motor Imagery EEG Classification." Sensors 20, no. 17 (2020): 4749. 